\title{
Positive predictive values for detection of trisomies 21,18 and 13 and termination of pregnancy rates after referral for advanced maternal age, first trimester combined test or ultrasound abnormalities in a national screening programme (2007-2009)
}

\author{
Jacqueline E. Siljee ${ }^{1 *}$, Alida C. Knegt ${ }^{2 \dagger}$, Maarten F. C. M. Knapen $^{3 \dagger}$, Mireille N. Bekker ${ }^{4 \dagger}$, Gerard. H. A. Visser $^{5}$ and Peter C. J. I. Schielen ${ }^{1}$ \\ ${ }^{1}$ Reference Laboratory for Pre- and Neonatal Screening/Laboratory for Infectious Diseases and Screening, National Institute for Public Health and the Environment, \\ Bilthoven, The Netherlands \\ 2Department of Clinical Genetics, University Medical Center Amsterdam, Amsterdam, The Netherlands \\ ${ }^{3}$ Department of Obstetrics and Prenatal Medicine, Erasmus MC Rotterdam, Stichting Prenatale Screening, Rotterdam, The Netherlands \\ ${ }^{4}$ Department of Obstetrics, Prenatal Diagnosis and Therapy, Radboud University Medical Center, Nijmegen, The Netherlands \\ ${ }^{5}$ Department of Obstetrics, University Medical Center Utrecht, The Netherlands \\ *Correspondence to: Jacqueline Siljee. E-mail: jacqueline.siljee@rivm.nl \\ ${ }^{\dagger}$ Also on behalf of the Working Group on Prenatal Diagnosis and Therapy.
}

\section{ABSTRACT}

Objective The objective of this article is to analyse the positive predictive value (PPV) of trisomies 21,18 and 13 after referral for advanced maternal age (AMA), first trimester combined test or ultrasound findings to suggest improvements for clinical practice.

Methods Data (48 457 combined tests, 134000 fetal anomaly scans and 24379 invasive prenatal tests) were combined to calculate PPV and termination of pregnancy rates.

Results For referral for AMA, the PPV for T21 was $1.0 \%$ and $1.8 \%$ for amniocentesis and chorionic villus biopsy, respectively; for the combined test at a maternal age $\geq 36$ years, these percentages were $4.9 \%$ and $12.5 \%$, respectively and for maternal age $<36$ years, $4.4 \%$ and $8.1 \%$, respectively. For ultrasound findings, the PPV was $5.3 \%$ and $14.8 \%$, respectively. Termination of pregnancy rate upon trisomy 21 diagnosis was $>90 \%$ unless detected after referral for ultrasound findings (71.5-85.9\%). About $50 \%$ of pregnant women with a high combined test risk chose not to have invasive testing.

Conclusions Advanced MA is still a large contributor to invasive testing but should be abandoned (low PPV, high fetal loss rate) and be replaced by reimbursable combined test screening for all women. Patient information on second trimester ultrasound screening should indicate that abnormal ultrasound findings are associated with high trisomy rate. (C) 2013 John Wiley \& Sons, Ltd.

Funding sources: None

Conflicts of interest: None declared

\section{INTRODUCTION}

Invasive prenatal diagnosis for fetal trisomies is usually based on advanced maternal age (AMA), first trimester screening or abnormal ultrasound findings. However, data on the positive predictive value (PPV) of these screening modalities and on the resulting incidence of termination of pregnancy (TOP) in case of a positive result are scattered. We previously addressed these issues in a regional study in The Netherlands. These studies showed that for referral for AMA, the PPV for trisomy 21 (T21) was 3\%; for the combined test, it was $10 \%$ and for second trimester ultrasound findings, it was $30 \% .{ }^{1}$ We also found that in this region, only $65 \%$ of all women with a high combined test risk chose for an invasive test. ${ }^{2}$

We now report on the complete nationwide Dutch data set from 2007 to 2009 to investigate PPV and TOP for AMA, combined test and ultrasound findings. For this purpose, we combined data from reports on first trimester combined test and second trimester anomaly scans within the Dutch prenatal screening programme and data of annual reports on invasive testing. On the basis of these findings, we provide suggestions to improve clinical practice. 


\section{MATERIAL AND METHODS}

\section{Organisation of the screening}

Invasive prenatal testing for AMA was implicated in the Dutch obstetric system since the early seventies. A more advanced national programme for prenatal screening, consisting of two tests, started in January 2007. The first is the combined test. This test was initially performed as a risk calculation specifically for the detection of T21 using maternal serum concentrations of pregnancy-associated plasma protein A and the free $\beta$-subunit of human chorionic gonadotrophin between 9 and $13+6$ weeks of gestational age (GA), combined with first trimester ultrasound measurement of the fetal nuchal translucency (NT) and maternal age (cut-off risk allowing invasive testing: 1 in 200). Risk calculation for trisomies 13 (T13) and 18 (T18) were only added in 2011. The second part of the programme consists of a fetal anomaly ultrasound scan between 18 and 22 weeks of GA. Informed decision and acknowledgement of the right not to be informed are hallmarks of the programme; the offer to be informed on the possibilities of prenatal screening is mandatory within the programme. When a woman decides to receive information on screening tests, she can opt in or out for either or both tests. Insurance companies reimburse the costs $(€ 155)$ for the ultrasound scan for women of all ages, whereas the first trimester combined test (€155) is reimbursed only for women of 36 years and older. Moreover, the latter group can still directly opt for invasive prenatal testing [i.e. amniocentesis (AC) or chorionic villus biopsy (CVS)], whereas younger women cannot. Invasive prenatal diagnosis and karyotyping in The Netherlands is by law strictly assigned to eight university medical centres, all with their assigned regions and together covering the entire country.

\section{Origin of data}

Complete annual data of the first trimester combined test (both demographic and pregnancy-related data, concentrations and MoM values of the biochemical parameters and NT measurements) were derived from the annual reports of the screening laboratories. ${ }^{3}$ Summarised data on the anomaly scan (numbers of anomaly scans in each region) were derived from an annual report. ${ }^{4}$ Complete annual data on invasive prenatal testing were derived from the 2006 to 2009 annual reports of the Working Group on Prenatal Diagnosis and Therapy (WPDT) of jointly the Dutch Association for Obstetrics and Gynaecology and the Dutch Society for Clinical Genetics.

\section{Study design}

Annual numbers of AC and CVS stratified for the reasons for referral were derived from the annual reports of the WPDT. The reasons for referral were (1) AMA, (2) high risk in combined test in women younger than 36 years, (3) high risk in combined test in women 36 years and older. Other referral categories were split up to produce a category for (4) ultrasound findings and (5) miscellaneous (consisting of five small referral categories; for example, previous chromosomal finding or neural tube defect in parents or siblings and previous aberrant genetic or biochemical result). Furthermore, the WPDT reports contain the results of the invasive tests in the referral categories AMA, combined test in women younger than 36 years, combined test in women 36 years and older and a referral category 'not AMA or combined test', which contains virtually all T21,T18 and T13 cases detected because of a referral after an abnormal ultrasound finding. Finally, the WPDT reports contained the numbers of pregnancies that were terminated after diagnosis. Our study reports on the results for T21, 18 and 13. Data were combined to determine the numbers of invasive tests performed (stratified for referral category and procedure; AC or CVS), PPV (expressed as the percentage of abnormal karyotype per invasive procedure performed), as well as the rates of TOP per referral category. To indicate statistically significant differences between referral categories or year, data were expressed as percentages with $95 \%$ confidence intervals (CIs).

Although only completely anonymised, accumulated and published data were used for this study, no approval by a medical ethical committee was needed.

\section{RESULTS}

To obtain a complete view on invasive testing after the start of the programme in 2007, a summary of data on invasive tests and referral categories is given in Table 1 . There was not a clear trend in the total number of invasive procedures between 2007 and 2009. Expressed as proportion of the total Dutch birth rate, however, there was a small decrease in $2009(4.2 \%$ compared with $4.5 \%$ in 2007-2008). Roughly two-thirds of the invasive tests consisted of AC, a percentage that did not change over time and varied between centres from $40 \%$ to $87 \%$ (data not shown).

The proportion of invasive testing for AMA decreased from $53.8 \%$ to $48.8 \%$ between 2007 and 2009, with a concomitant small increase of the proportion of referrals for combined test (from $16.3 \%$ to $18.9 \%$ ) and ultrasound findings (from $16.7 \%$ to $18.9 \%)$. Referrals for ultrasound findings were followed by an AC (increasing from $67.2 \%$ to $72.8 \%$ ) or by a CVS (decreasing from $32.8 \%$ to $27.2 \%$ ).

Table 2 summarises the PPV per referral category for 2007 to 2009, for T21 and all major trisomies combined (T21, T18 and T13). Referrals based on AMA followed by AC had a fourfold lower chance of showing a case of T21 compared with referrals based on combined test screening for women $\geq 36$ years of age or ultrasound findings (PPV: $1.0 \%, 4.9 \%$ and $5.3 \%$, respectively). For CVS, these differences were even more pronounced; AMA was about seven times less effective than combined test screening and about nine times less effective than ultrasound abnormalities (PPV 1.8\%, 12.5\% and 14.8\%, respectively). For T21, T18 and T13 combined, the PPV after CVS for AMA, combined test $\geq 36$ years of age and ultrasound findings were $2.6 \%, 17.3 \%$ and $29.2 \%$, respectively. The PPV after referral for combined test was in general higher for the age group $\geq 36$ years as compared with $<36$ years. Notably, the PPV to detect a T21, T18 or T13 after referral for ultrasound findings (12.7\% after AC and $29.2 \%$ after CVS) was about twice the PPV after referral for combined screening $\geq 36$ years $(6.0 \%$ and 
Table 1 Number of invasive tests per referral category and type of invasive test [amniocentesis (AC) or chorionic villus biopsy (CVS)] in The Netherlands, 2007 to 2009

\begin{tabular}{|c|c|c|c|c|}
\hline & 2007 & 2008 & 2009 & 2007-2009 \\
\hline Birth rate ${ }^{a}$ & 181336 & 184634 & 184915 & 550885 \\
\hline Total number of invasive tests (\% of birth rate, Cl) & $\begin{array}{c}8225 \\
4.5(4.4,4.6)\end{array}$ & $\begin{array}{c}8341 \\
4.5(4.4,4.6)\end{array}$ & $\begin{array}{c}7813 \\
4.2(4.1,4.3)\end{array}$ & $\begin{array}{c}24379 \\
4.4(4.4,4.4)\end{array}$ \\
\hline Number of $\mathrm{AC}(\% ; \mathrm{Cl})$ & $\begin{array}{c}5487 \\
66.7(65.7,67.7)\end{array}$ & $\begin{array}{c}5525 \\
66.2(65.2,67.2)\end{array}$ & $\begin{array}{c}5183 \\
66.3(65.3,67.4)\end{array}$ & $\begin{array}{c}16195 \\
66.4(65.8,67)\end{array}$ \\
\hline Number of CVS $(\% ; \mathrm{CI})$ & $\begin{array}{l}2738 \\
33.3(32.3,34.3)\end{array}$ & $\begin{array}{c}2816 \\
33.8(32.8,34.8)\end{array}$ & $\begin{array}{c}2630 \\
33.7(32.6,34.7)\end{array}$ & $\begin{array}{c}8184 \\
33.6(33,34.2)\end{array}$ \\
\hline \multicolumn{5}{|l|}{ Referral for ( $\mathrm{N}$ and as \% of total number of $\mathrm{AC} / \mathrm{CVS}$ ) } \\
\hline Maternal age & $\begin{array}{c}4424 \\
53.8(52.7,54.9)\end{array}$ & $\begin{array}{c}4259 \\
51.1(50,52.1)\end{array}$ & $\begin{array}{c}3812 \\
48.8(47.7,49.9)\end{array}$ & $\begin{array}{c}12495 \\
51.3(50.6,51.9)\end{array}$ \\
\hline $\mathrm{AC}(\% ; \mathrm{Cl})$ & $\begin{array}{c}3126 \\
70.7(69.3,72)\end{array}$ & $\begin{array}{c}2962 \\
69.5(68.1,70.9)\end{array}$ & $\begin{array}{c}2648 \\
69.5(68,70.9)\end{array}$ & $\begin{array}{c}8736 \\
69.9(69.3,70.9)\end{array}$ \\
\hline CVS $(\% ; C l)$ & $\begin{array}{c}1298 \\
29.3(28,30.7)\end{array}$ & $\begin{array}{c}1297 \\
30.5(29.1,31.9)\end{array}$ & $\begin{array}{c}1164 \\
30.5(29.1,32)\end{array}$ & $\begin{array}{c}3759 \\
30.1(29.3,30.9)\end{array}$ \\
\hline Combined test age $\geq 36$ years & $\begin{array}{c}717 \\
8.7(8.1,9.3)\end{array}$ & $\begin{array}{c}777 \\
9.3(8.7,10)\end{array}$ & $\begin{array}{c}789 \\
10.1(9.4,10.8)\end{array}$ & $\begin{array}{c}2283 \\
9.4(9,9.7)\end{array}$ \\
\hline $\mathrm{AC}(\% ; \mathrm{Cl})$ & $\begin{array}{c}492 \\
68.6(65.1,71.9)\end{array}$ & $\begin{array}{c}510 \\
65.6(62.2,68.9)\end{array}$ & $\begin{array}{c}533 \\
67.6(64.2,70.7)\end{array}$ & $\begin{array}{c}1535 \\
67.2(65.3,69.1)\end{array}$ \\
\hline CVS $(\% ; C l)$ & $\begin{array}{c}225 \\
31.4(28.1,34.9)\end{array}$ & $\begin{array}{c}267 \\
34.4(31.1,37.8)\end{array}$ & $\begin{array}{c}256 \\
32.4(29.3,35.8)\end{array}$ & $\begin{array}{c}748 \\
32.8(30.9,34.7)\end{array}$ \\
\hline Combined test age $<36$ years & $\begin{array}{c}625 \\
7.6(7,8.2)\end{array}$ & $\begin{array}{c}695 \\
8.3(7.8,8.9)\end{array}$ & $\begin{array}{c}687 \\
8.8(8.2,9.4)\end{array}$ & $\begin{array}{c}2007 \\
8.2(7.9,8.6)\end{array}$ \\
\hline$A C(\% ; C)$ & $\begin{array}{c}400 \\
64.0(60.2,67.7)\end{array}$ & $\begin{array}{c}450 \\
64.7(61.1,68.2)\end{array}$ & $\begin{array}{c}423 \\
61.6(57.9,65.1)\end{array}$ & $\begin{array}{c}1273 \\
63.4(59.5,63.7)\end{array}$ \\
\hline CVS $(\% ; \mathrm{Cl})$ & $\begin{array}{c}225.00 \\
36(32.3,39.8)\end{array}$ & $\begin{array}{c}245.00 \\
35.3(31.8,38.9)\end{array}$ & $\begin{array}{c}264.00 \\
38.4(34.9,42.1)\end{array}$ & $\begin{array}{c}734.00 \\
36.6(34.5,38.7)\end{array}$ \\
\hline Ultrasound findings & $\begin{array}{c}1377 \\
16.7(16,17.6)\end{array}$ & $\begin{array}{c}1550 \\
18.6(17.8,19.4)\end{array}$ & $\begin{array}{c}1465 \\
18.8(17.9,19.6)\end{array}$ & $\begin{array}{c}4392 \\
18(17.5,18.5)\end{array}$ \\
\hline $\mathrm{AC}(\% ; \mathrm{Cl})$ & $\begin{array}{c}925 \\
67.2(64.7,69.6)\end{array}$ & $\begin{array}{c}1097 \\
70.8(68.5,73)\end{array}$ & $\begin{array}{c}1066 \\
72.8(70.4,75)\end{array}$ & $\begin{array}{c}3088 \\
70.3(68.9,71.6)\end{array}$ \\
\hline CVS $(\% ; \mathrm{Cl})$ & $\begin{array}{c}452 \\
32.8(30.4,35.3)\end{array}$ & $\begin{array}{c}453 \\
29.2(27,31.5)\end{array}$ & $\begin{array}{c}399 \\
27.2(25,29.6)\end{array}$ & $\begin{array}{c}1304 \\
29.7(28.4,31.1)\end{array}$ \\
\hline Miscellaneous & $\begin{array}{c}1082 \\
13.2(12.4,13.9)\end{array}$ & $\begin{array}{c}1060 \\
12.7(12,13.4)\end{array}$ & $\begin{array}{c}1060 \\
13.6(12.8,14.3)\end{array}$ & $\begin{array}{c}3202 \\
13.1(12.7,13.6)\end{array}$ \\
\hline $\mathrm{AC}(\% ; \mathrm{Cl})$ & $\begin{array}{c}544 \\
50.3(47.3,53.3)\end{array}$ & $\begin{array}{c}506 \\
47.7(44.7,50.7)\end{array}$ & $\begin{array}{c}513 \\
48.4(45.4,51.4)\end{array}$ & $\begin{array}{c}1563 \\
48.8(47.1,50.5)\end{array}$ \\
\hline CVS $(\% ; \mathrm{Cl})$ & $\begin{array}{c}538 \\
49.7(46.7,52.7)\end{array}$ & $\begin{array}{c}554 \\
52.3(49.3,55.3)\end{array}$ & $\begin{array}{c}547 \\
51.6(48.6,54.6)\end{array}$ & $\begin{array}{c}1639 \\
51.2(49.5,52.9)\end{array}$ \\
\hline
\end{tabular}

$\mathrm{Cl}$, confidence interval.

Data in the table are absolute numbers and proportions with $95 \% \mathrm{Cl}$.

The composition of the category 'miscellaneous' is described in the Materials and Methods Section.

airth rates are from Statistics Netherlands (www.cbs.nl., accessed 3/8/13).

$17.3 \%$, respectively). In all categories, the PPV for trisomies was two to three times higher in the CVS group as compared with AC.

T21 TOP rates were nearly $90 \%$ or higher for all categories of referrals except for ultrasound findings, where the TOP rate was distinctively lower (Table 3). There were too few cases of $\mathrm{T} 18$ and $\mathrm{T} 13$ to draw conclusions from.

\section{DISCUSSION}

In this study, we combined the data of 3 years of screening in The Netherlands after the introduction of a new prenatal screening policy by evaluating PPV of various referral categories and data on TOP after invasive testing. It is of interest that the introduction of the nationwide screening programme in The Netherlands in 2007 hardly resulted in changes in absolute or relative numbers per referral category for invasive testing (Table 1). During this study period, T18 and T13 risks were not yet reported as part of the combined test, but even so, the combined test picked up a fair number of T18 and T13 cases. This is in agreement with our previous report on T13 and T18 detection with the combined test. ${ }^{5}$

The percentage of invasive tests because of AMA alone remained high between 2007 and 2009 (Table 1). However, 
Table 2 Positive predictive value (and confidence interval) for T21 and for T13, T18 and T21 combined after amniocentesis (AC) or chorionic villus biopsy (CVS) for 2007 to 2009 combined

\begin{tabular}{llcc} 
Referral for & & T21 & T21-18-13-combined \\
\hline Maternal age & AC & $1.0(0.8-1.2)$ & $1.3(1.1-1.6)$ \\
\hline $\begin{array}{c}\text { Combined test age } \\
\geq 36 \text { years }\end{array}$ & AC & $1.8(1.4-2.2)$ & $2.6(2.1-3.1)$ \\
\hline $\begin{array}{c}\text { Combined test age } \\
<36 \text { years }\end{array}$ & AC & $12.0-6.1)$ & $6.0(4.9-7.3)$ \\
\hline & CVS & $8.1(3.4-5.6)$ & $4.7(3.7-6.0)$ \\
\hline Ultrasound findings & AC & $5.3(4.6-6.2)$ & $10.8(8.8-13.3)$ \\
\hline & CVS & $14.8(13.0-16.8)$ & $29.2(26.9-31.7)$ \\
\hline
\end{tabular}

the incidence of diagnosed cases of T21 with an AP was fourfold and with a CVS was up to sevenfold lower that of referral for a combined test high risk (Table 2). Published data on the comparison of PPV for AMA and combined test in routine screening programmes are scarce, but these data are in agreement with those or an earlier regional study in The Netherlands. ${ }^{1}$ We previously have reported on the costs and effects of various screening scenarios in The Netherlands. ${ }^{6}$ Although a detailed cost-effectiveness analysis is beyond the scope of this article, a rough estimate learns that replacing all AMA by combined tests would save $75 \%$ of the costs. The detected fetal loss to detected T21 case rate would be one loss for every two detected for AMA referral and 1 loss for every 15 detected for the combined test. So, there are many reasons to abandon screening on the basis of AMA. In anticipation of the notion that referral for AMA is suboptimal, the coordinating committee of the Dutch Down syndrome screening programme did send an advice to the Minister of Health in December 2011 to abolish AMA as an indication for invasive testing and to make the costs of the combined test reimbursable for women younger than 36 years as well.

The PPV for aneuploidies at karyotyping after AC or CVS after referral for abnormal ultrasound findings were the highest of all screening tests; the PPV to pick up any trisomy after a referral for abnormal ultrasound findings was $29.2 \%$, so nearly one out of three (Table 2). A relatively small fraction of referrals for ultrasound findings may have consisted of anomalies discovered in the first trimester, mainly because of the fact that a few patients were referred immediately after a large NT measurement, without performing a serum analysis for the combined test. The vast majority of all referrals for abnormal ultrasound findings were, however, because of an 18 to 22-week screening anomaly scan (which was performed in at least $80 \%$ of the pregnant population in 2009) and about 15 000-s expert structural anomaly scans for enhanced risk for fetal structural anomalies. ${ }^{4}$ Also, in our previous study concerning a regional screening programme, ultrasound examinations were mostly second trimester scans. ${ }^{1}$

The anomaly scan was introduced in the Dutch screening programme as a screening test for structural anomalies, specifically neural tube defects, not as a screening test for aneuploidies. The data of this study show however that the anomaly scan, both in terms of PPV and absolute numbers, is

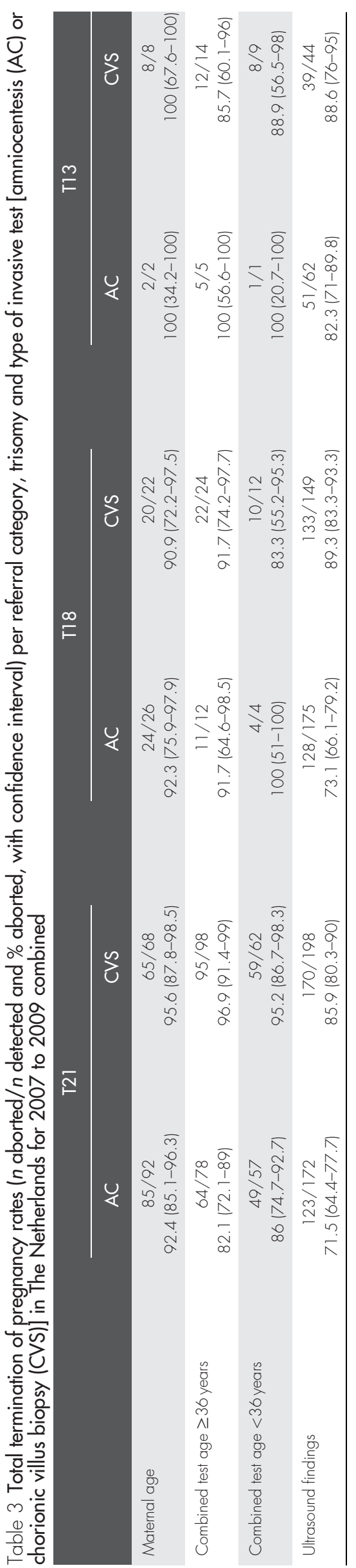

(c) 2013 John Wiley \& Sons, Ltd. 
superior to the combined test to pick up T21, T18 and T13. This does not imply that the anomaly scan is a good screening test for T21, as we do not know the number of T21 cases missed by the anomaly scan, nor do we know how many pregnant women with abnormal ultrasound findings opt out for invasive prenatal diagnosis. Presently, the patient information leaflet does not mention that many genetic abnormalities are discovered by the anomaly scan. On the basis of the present data, we advise to counsel an abnormal karyotype, especially the common aneuploidies, as an unexpected finding.

In a previous report on TOP rates for detected trisomies in The Netherlands between 1991 and 2000, 88\% of T21, 79\% of T18 and $81 \%$ of T13 pregnancies were terminated. ${ }^{7}$ A study reviewing a large cohort of the UK data also showed TOP rates for T21 pregnancies of $81 \% .^{8}$ A recent Canadian study showed a TOP rate of $66.7 \%$ and included a review of the literature showing a TOP rate of $70 \%$ to $100 \% .^{9}$ The TOP rates of the current study generally match both the historical Dutch data as well as the data from other countries. It appeared that TOP rates after AC because of referral for ultrasound findings were lowest of all. This could be due to the fact that the anomaly scan was aimed at detecting ultrasound abnormalities and not at detecting aneuploidies as a primary outcome, being 'unexpected findings'. Additionally, these TOP may be pushed too much towards the legally accepted GA for TOP in The Netherlands (until 23 weeks and 6 days) as well. Both facts might not warrant TOP for this subgroup of pregnant women. Other reasons, for example, emotional reasons and the expectancy of the natural demise of these pregnancies, could also play a role. A higher uptake of first trimester screening may well avoid some of these problems.

The percentage of high-risk results for the Down syndrome screening in 2009 was $6.2 \%$ (CI: $5.9-6.5 \%$ ) of a total of 48457 tests $^{3}$ or 3005 (CI: 2854-3161) referrals. The actual number of invasive tests was only 1476 (Table 1). This indicates that about $50 \%$ of pregnant women with high-risk results after a combined test still decide to opt out for a diagnostic test. This is in line with the previously published $65 \%$ opting out in a regional Dutch population ${ }^{2}$ and with $49.3 \%$ in a recent Canadian study. ${ }^{9}$ A detailed analysis of which women with high risks opt out for invasive testing, possibly explaining part of this phenomenon, was published recently. ${ }^{2}$

The strength of this article is the large and nationwide data set. A shortcoming is the inability to exactly quantify the number of first versus second trimester referrals for abnormal ultrasound findings. Moreover, the total number of referrals for abnormal ultrasound findings was not completely clear in all cases, because we assumed that among the referrals other than combined test or AMA, reported T21,T18 and T13 cases were mostly from referrals for ultrasound findings (other referral categories were shown to detect virtually no T21, T18 or T13). It would be of interest to analyse the results of all referral categories in relation to the MA at prenatal diagnostic test, but these data are currently not available. Thus, there is still room for improvement in registration.

In conclusion, invasive prenatal diagnosis based on AMA alone is still a large contributor to invasive testing. However, given its low PPV, high fetal loss rate and high costs, this indication should be abandoned and be replaced by first trimester screening free of charge for all women. The presence of fetal abnormalities at second trimester ultrasound scan had the highest detection rate of trisomy but is unlikely to replace first trimester screening because false negatives are unknown, because trisomy detection is currently not the main reason for such a scan and because a diagnosis is usually made rather late.

\section{ACKNOWLEDGEMENTS}

We thank all the contributors to the annual reports of the WPDT (and especially N. de Man), the representatives of the screening laboratories and the representatives of the regional screening organisations who gathered the data for the annual reports.

Screening laboratories: Departments of Clinical Chemistry of the VU university Medical Centre (Dr A. Heijboer), the Academic Medical Centre Amsterdam (Prof. Dr A. Sturk), the Maastricht University Medical Centre (Dr P.P. Menheere), the Rijnstate Hospital Arnhem (Dr D. Telting, Dr P.M.W. Janssens), the University Medical Centre Groningen (Dr A.C. MullerKobold) and the Star Medical Diagnostics Centre, Rotterdam (Dr Y. de Rijke, Dr J.A.M. Laudy).

Regional prenatal screening organisations: Northern region (Prof. Dr. C.M. Bilardo), Centre region (Dr Ph. Stoutenbeek), Southern region (Dr A.B.C. Coumans), two Northern Holland regions including Amsterdam (Dr E. Pajkrt, Dr M. Engels), Eastern region (Dr A.M. Stolwijk), South-western region (Drs J.C.I.Y Reijerink-Verheij, MHA, Dr M.F.C.M. Knapen) and Northern South Holland region (Prof. Dr. D. Oepkes).

Clinical genetics Centres or departments for prenatal diagnostics of the Albert Schweitzer Hospital Dordrecht, Academic Medical Centre Amsterdam, Amphia Hospital Breda, Maastricht University Medical Centre, Diagnostic Centre Eindhoven, Erasmus University Medical Centre Rotterdam, Isala Clinics Zwolle, Jeroen Bosch Hospital Den Bosch, Leiden University Medical Centre, Medical Centre Haaglanden The Hague, Medical Centre Leeuwarden, Medical Centre Twente Enschede, Reinier the Graaf Hospital Delft, Rijnstate Hospital Arnhem, St. Elisabeth Hospital Tilburg, University Medical Centre Groningen, University Medical Centre St Radboud Nijmegen, Utrecht University Medical Centre and Free University Medical Centre Amsterdam.

We also thank Ms Z. Schielen for her assistance with data processing.

\section{WHAT'S ALREADY KNOWN ABOUT THIS TOPIC?}

- Reports on positive predictive value and termination of pregnancy rates of nationwide routine screening programmes do exist but are scarce.

\section{WHAT DOES THIS STUDY ADD?}

- This is one of the very few reports on an entire nationwide screening programme that presents data on positive predictive value and termination of pregnancy rates after referral for advanced maternal age, first trimester combined test and referral for ultrasound findings. This study provides evidence that screening for advanced maternal age should be abandoned. 


\section{REFERENCES}

1. Lichtenbelt KD, Alizadeh BZ, Scheffer PG, et al. Trends in the utilization of invasive prenatal diagnosis in The Netherlands during 2000-2009. Prenat Diagn2011;31:765-72.

2. Lichtenbelt KD, Schuring-Blom GH, van der Burg N, et al. Factors determining uptake of invasive testing following first-trimester combined testing. Prenat Diagn 2013;33:328-33.

3. Schielen PCJI. Quality control parameters of Dutch Down's syndrome screening laboratories 2009 (2007-2008, when available). 2011. RIVM report 230083002/2011. (http://www.rivm.nl/dsresource? objectid=rivmp:119025\&type=org\&disposition=inline\&ns_nc=1).

4. Fracheboud J, Van Agt HME, De Koning, HJ. Monitoring 2010. Report: Screeningsprogramma Downsyndroom/Structureel echoscopisch onderzoek. Erasmus University Rotterdam, the Netherlands (In Dutch). 2012. (http://www.rivm.nl/dsresource?objectid=rivmp:193263\&type =org\&disposition=inline).
5. Kuc S, Koster MP, Visser GH, et al. Performance of first-trimester serum screening for trisomy 21 before and from $11+0$ weeks of gestational age in The Netherlands. Prenat Diagn 2010;30:906-8.

6. Hoogendoorn, M, Evers, SMAA, Schielen PCJI, et al. Costs and effects of prenatal screening methods for Down syndrome and neural tube defects. Community Genet 2008;11:359-67.

7. Nagel HT, Knegt AC, Kloosterman MD, et al. Invasive prenatal diagnosis in The Netherlands, 1991-2000: number of procedures, indications and abnormal results detected. Ned Tijdschr Geneeskd 2004;148:1538-43.

8. Irving C, Basu A, Richmond S, et al. Twenty-year trends in prevalence and survival of Down syndrome. Eur J Hum Genet 2008;11:1336-40.

9. Metcalfe A, Currie G, Johnson JA, et al. Impact of observed versus hypothesized service utilization on the incremental cost of first trimester screening and prenatal diagnosis for trisomy 21 in a Canadian province. Prenat Diagn 2013;33:429-35. 